\title{
Demut gegenüber der Fehlbarkeit des Handelns im Business-Coaching (Humility in the face of the fallibility of action in business coaching)
}

\author{
Michael Scherf
}

Angenommen: 7. Juli 2021 / Online publiziert: 4. August 2021

(C) Der/die Autor(en) 2021

Heidi Möller, Jannik Zimmermann (2020): Schwierige Situation im BusinessCoaching. Praxisbeispiele, Perspektiven und Handlungsmöglichkeiten. Wiesbaden: Springer, 206 Seiten, 39,99€.

Welchen herausfordernden Situationen begegnen Coaches in ihrem Berufsalltag? Welche Sichtweisen auf diese Situationen sind ertragreich und zeigen Umgangsweisen mit diesen typischen Alltagsherausforderungen auf? Dieses Buch illustriert anhand von 31 Fallbeschreibungen als ,schwierig“ erlebte Situationen im Coaching und kommentierende Einordnungen unterschiedlicher erfahrener Coaches, was diese Tätigkeit prägt und welche Fallstricke sie bereithält. Das Werk trägt somit dazu bei, die immer noch bestehende Lücke zwischen der marketingorientierten Schauseite des Coachings und dem um die falladäquate Bewältigung von praktischen Herausforderungen ringenden Beratungsalltag zu schließen.

Die hier präsentierten Kurzbeschreibungen von tatsächlichen Handlungssituationen aus der Coachingspraxis stammen aus einer retrospektiven Onlinebefragung von Coaches zu Situationen, die diese selbst als „schwierig“ wahrgenommen haben. Die Ergebnisse wurden dann Kategorien zugeordnet (z.B. „Verhalten der Coachees“, „Erwartungen der Coachees an die Coaches“ oder auch „Einfluss (aus) der beauftragenden Organisation"), die die Gliederung des Buches ergeben. Die Fälle wurden in mehreren Gruppendiskussionen von namentlich gekennzeichneten erfahrenen Coaches kommentiert und den jeweiligen Fällen als komprimierte Verschriftlichungen beigefügt.

Das Buch erinnert an mehrperspektivische Fallsupervision und schließt damit z.B. an Billmeier et al. (2005) an, die schon 2005 Fallvignetten über die Ausgangssituationen von Coachingsprozessen, kommentiert durch bekannte Coaches, veröf-

Dr. Michael Scherf $(\triangle)$

Brüder-Grimm-Str. 60, 34134 Kassel, Deutschland

E-Mail: Michael-Scherf@gmx.de

Universität Kassel, Kassel, Deutschland 
fentlicht haben. Coaching wird im vorliegenden Buch in beeindruckender Weise als Berufsfeld sichtbar, das zwischen den Anforderungen der heldenhaften Kompetenzdarstellungskompetenz (Pfadenhauer 2003) und einer eher handwerklichen mit Mehrdeutigkeit und Unsicherheit ringenden Handlungspraxis aufgespannt ist. Professionssoziologisch haben wir es also mit einer potenziell professionalisierungsbedürftigen Praxis zu tun (z. B. Oevermann 2003; Schütze 1994; Scherf 2010).

In den hier vorliegenden Fallbeschreibungen werden die Chancen und Herausforderungen, sich selbstkritisch und wissenschaftlich in diesem Feld zu bewegen, beeindruckend aufgezeigt:

- Die Fallgeber/innen schildern nicht selten Situationen, die nur erahnen lassen, welcher Schwierigkeit sie sich überhaupt ausgesetzt sahen. Sie tun sich also häufig sichtbar schwer damit, sich als herausgefordert darzustellen. Unsicherheit und mögliche „Fehler“ scheinen nicht zum üblichen Darstellungsrepertoire dieses Berufsstandes zu gehören.

- Hier und da geraten auch die Kommentare der erfahrenen Coaches auf den Pfad der Darstellung der eigenen Kompetenz, nicht mehr ganz fokussiert auf das Verstehen der Hausforderung der Fallgeber/innen.

- Die Arbeit mit authentischen Primärdaten bleibt eine besonders lohnenswerte wissenschaftliche Herausforderung. Eine spannende Weiterführung der Idee dieses Buches wäre z. B. der audiovisuelle Mitschnitt realer Beratungsgespräche mit direkt anschließender Kommentierung über das Erleben aus Sicht von Coach, aber besonders auch von Coachee (vgl. Möller 1998). Viel zu oft gehen wir ja davon aus, dass es der Coach quasi allein ist, der eine Situation handelnd meistert, und übersehen die Leistungen der Klient/innen, ebenfalls mit „schwierigen“ Situation umgehen zu müssen.

Für Praktiker/innen, aber gerade auch für angehende Coaches, ist dieses Buch eine wunderbare Fundgrube, die Reflexion der eigenen Praxis weiter zu trainieren und eine gewisse Demut gegenüber der Fehlbarkeit des Handelns zu behalten. Jeder Kommentar und jede Falldarstellung zeigt die enge Verwobenheit von theoretischer Orientierung und praktischer Ableitung/Handlung transparent auf. Die mehrfache Kommentierung der Fallschilderungen macht deutlich, wie jeder Akteur mit seiner individuellen Rekonstruktion der geschilderten Situation bestimmte Zusammenhänge hell beleuchtet und damit andere eher in den Schatten gestellt werden. Diese Mehrfachausleuchtung der Szenerie bildet sich auch im Aufbau des Buches durch die direkte Folge mehrerer Kommentare zu einem Fall sehr gut ab. Dieses eher handwerkliche Abwägen ist Praktiker/innen als innere Haltung zu wünschen, gerade in einem Feld, das nach strahlenden Helden und dem Wunsch nach Eindeutigkeit und Sicherheit giert.

Funding Open Access funding enabled and organized by Projekt DEAL.

Open Access Dieser Artikel wird unter der Creative Commons Namensnennung 4.0 International Lizenz veröffentlicht, welche die Nutzung, Vervielfältigung, Bearbeitung, Verbreitung und Wiedergabe in jeglichem Medium und Format erlaubt, sofern Sie den/die ursprünglichen Autor(en) und die Quelle ordnungsgemäß nennen, einen Link zur Creative Commons Lizenz beifügen und angeben, ob Änderungen vorgenommen wurden. 
Die in diesem Artikel enthaltenen Bilder und sonstiges Drittmaterial unterliegen ebenfalls der genannten Creative Commons Lizenz, sofern sich aus der Abbildungslegende nichts anderes ergibt. Sofern das betreffende Material nicht unter der genannten Creative Commons Lizenz steht und die betreffende Handlung nicht nach gesetzlichen Vorschriften erlaubt ist, ist für die oben aufgeführten Weiterverwendungen des Materials die Einwilligung des jeweiligen Rechteinhabers einzuholen.

Weitere Details zur Lizenz entnehmen Sie bitte der Lizenzinformation auf http://creativecommons.org/ licenses/by/4.0/deed.de.

\section{Literatur}

Billmeier, R., et al. (2005). Der Beginn von Coachingprozessen. Vom Fall zum Konzept. Bergisch Gladbach: EHP.

Möller, H. (1998). Selbstkonfrontationsinterviews bei Experten der Supervision. Zum Design einer interaktionszentrierten Supervisionsforschung. In P. Berker \& F. Buer (Hrsg.), Praxisnahe Supervisionsforschung. Felder, Designs, Ergebnisse (S. 196-215). Münster: Votum.

Oevermann, U. (2003). Kodifiziertes Wissen und persönliche Erfahrung in der professionalisierten Praxis stellvertretender Krisenbewältigung. In J. Fried \& T. Kailer (Hrsg.), Wissenskulturen. Beiträge zu einem forschungsstrategischen Konzept (S. 195-210). Berlin: Akademie Verlag.

Pfadenhauer, M. (2003). Professionalität: Eine wissenssoziologische Rekonstruktion institutionalisierter Kompetenzdarstellungskompetenz. Opladen: Leske + Budrich.

Scherf, M. (2010). Strukturen der Organisationsberatungsinteraktion. Objektiv hermeneutische Untersuchung zur Professionalisierungsbedürftigkeit der Organisationsberatung. Göttingen: Vandenhoeck \& Ruprecht.

Schütze, F. (1994). Strukturen des professionellen Handelns, biographische Betroffenheit und Supervision. Supervision, 26, 10-39. 